\title{
Noble Ganga Tears-Sweat Bomb Theory
}

\author{
Thiyagarajan Babu*
}

\begin{abstract}
Tears and perspiration are the regular secretions of eye organ for any human being owing to emotional issues. There were numerous literatures available on the products associated with the tears, why tears were created but the how part was not solved till date. Huge volume of literatures projecting tears were secreted by the lachrymal glands involving complex body mechanism was available. Similarly the roles of sweat glands were attributed for perspiration in the skin of mammals. My simple two instruments namely pH meter and ORP (Oxidation Reduction Potential) meter unopened this mystery that tears and perspiration were formed with an outside molecule. Lachrymal glands and Sweat glands secreted vital compounds carried by the cellular fluid in order to protect eyes and skin respectively from harmful organisms or agents.
\end{abstract}

\section{(I)TEAR FILM}

\section{Introduction}

Human beings consume water and lachrymal gland secretion adheres to the tear film (Wu K, Galina VJ, 2006). The normal water was converted as cellular water in order to reduce the surface tension of the water to lubricate the cornea and conjunctiva, to provide anti-bacterial activity, to keep the cornea moist, to serve as a conduit for the entry of polymorphonuclear leucocytes into the cornea to remove toxic substances from the ocular surface for different protection of the eyes. They were not tears as the surface tension of cellular water and the tears were varied.

\section{(ii)HYDROGEN- THE WONDER MOLECULE}

Hydrogen is the lightest element and its monoatomic form $\left(\mathrm{H}_{1}\right)$ is the most abundant chemical substance (75 per cent of the Universe's baryonic mass (Finkel T, Holbrook 2000). $\mathrm{H}_{2}^{+}$is composed of two positively charged protons and one negatively charged electron and lack electron-electron repulsion. Being the lightest gas Hydrogen diffuses in the cells as rapidly as possible. Notice that the hydrogen bond is somewhat longer than the covalent $\mathrm{O}-\mathrm{H}$ bond. This means that it is considerably weaker; it is so weak, in fact, that a given hydrogen bond cannot survive for more than a tiny fraction of a second. This build up more and more Hydrogen molecules pressure in the cell in addition to Hydrogen's released due to internal and external pressures.

About two-thirds of this water is located within cells, while the remaining third consists of extracellular water, mostly in the blood plasma and in the interstitial fluid that bathes the cells. The water molecules are made up of tiny combination of three nuclei and eight electrons and have unique properties. This would ordinarily result in a tetrahedral geometry in which the angle between electron pairs (and therefore the $\mathrm{H}-\mathrm{O}-\mathrm{H}$ bond angle) is $109.5^{\circ}$. However, because the two non-bonding pairs remain closer to the oxygen atom, these exert a stronger repulsion against the two covalent bonding pairs, effectively pushing the two hydrogen atoms closer together. The result is a distorted tetrahedral arrangement in which the $\mathrm{H}-\mathrm{O}-\mathrm{H}$ angle is $104.5^{\circ}$ (Lide, David R, 2003).

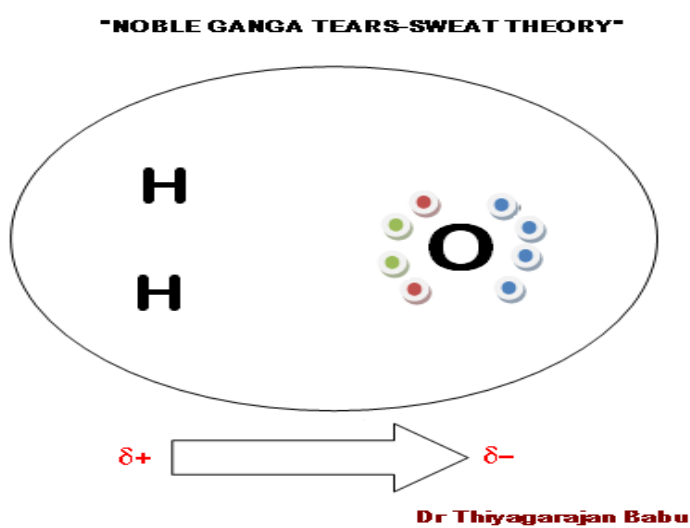

In the human system the biological activity of proteins (of which enzymes are an important subset) is critically dependent not only on their composition but also on the way these huge molecules are folded; this folding involves hydrogen-bonded interactions with water, and also between different parts of the molecule 
itself. Anything that disrupts these intra-molecular hydrogen bonds will denature the protein and destroy its biological activity (Source: Water Structure and Properties).

It is worth mentioning that the source of Hydrogen molecules were not only the hydrogen-bonding always involved with water; plus the two parts of the DNA double helix are held together by $\mathrm{H}-\mathrm{N}-\mathrm{H}$ hydrogen bonds (Greenwood, Norman N.; Earnshaw, 1997).

Hence, there is a huge source of Hydrogen molecules exist in the human system.

The present thinking, influenced greatly by molecular modeling simulations beginning in the 1980s, is that on a very short time scale (less than a picoseconds), water is more like a "gel" consisting of a single, huge hydrogenbonded cluster. On a 10-12-10-9 sec time scale, rotations and other thermal motions cause individual hydrogen bonds to break and re-form in new configurations, inducing ever-changing local discontinuities whose extent and influence depends on the temperature and pressure (Source: www.imimphd.edu.pl/contents/Lectures/Electrochemistry). Hence, the Hydrogen molecules were prone for diffusion when there were pressures in the cells besides their weak property due to its lightest nature unlike other stable molecules like Oxygen. Those millions of Hydrogen molecules diffuse through the tissues like bullets in search of an exit in the human system.

\section{Thousands Years of Cry on Cry}

Molecules have quantized energy levels that can be analyzed by detecting the molecule's energy exchange through absorbance or emission (Nic, M.; Jirat, J.; Kosata, B., 2006). Whenever there was a pressure of any kind internal or external, the billions of human cells were pressurized and this led to many molecule's energy exchange through absorbance or emission or and human physio-chemical alterations.

\section{(i) Eyes and Tear Mechanism}

Eyes were prone for all kinds of pressures both internally and environmentally. Once there was a pressure that had to be released which was a natural law. Strain in eye region inevitably pressurizes the tissues around and that triggers the release of millions of Hydrogen molecules. The lightest Hydrogen molecules were fired like bullets from different tissues and march like a battalions in search of an exit. The exit being the eyes inner layer they make a cloud of Hydrogen molecules being positively charged. Why does Hydrogen released like bullets? "Hydrogen Bomb" was created by uniting the two Hydrogen molecule nucleuses. If such things happen in human cell then cells would bulge and tear away. Hence the powerful neurotransmitters signal the brain instantly to rush the hydrogen molecules out of cells as fast as possible in order to avoid any collision of two hydrogen molecules. Released Hydrogen molecules due to various pressure, "stick" to the surface of the tear film moving through it. This layer of Hydrogen molecules forms the "Hydrogen cloud" at the the innere layer of eyes. At the surface of the eyes, it is essentially static due to the friction of the surface. The Hydrogen molecules, with its boundary layer were in fact the new shape of the eyes that the rest of the molecules form the new water molecules with the atmospheric Oxygen. This boundary layer could separate from the surface of the eyes' inner layer, essentially creating a new surface and completely changing the flow path of tears. In general, the liquids drift to the bottom upon mixing hence the cellular water and the tears which were formed outside the tear film mix together. And this was the reason many scientists reported tears with innumerable products.

They get the contact of atmospheric stable Oxygen which was electromagnetic negatively charged molecules. The first tear molecule was formed with a plucking feeling in the human eyes. This led to further bonding of positively charged Hydrogen molecules with negatively charged Oxygen molecules. The chain reaction continues and the tears start shedding due to gravitational pull. As the drops get bigger, their weight deforms them into the typical tear shape.

Oxygen as a compound is present in the atmosphere in trace quantities in the form of carbon dioxide $\left(\mathrm{CO}_{2}\right)$. Due to its electro-negativity, oxygen forms chemical bonds with almost all other elements at elevated temperatures to give corresponding oxides.

I just compare this process with the process of rain drop lets formation in the sky. There was no sac like lachrymal gland in the sky in order to secrete huge water molecules in order to rain. The atmospheric Hydrogen and Oxygen molecules get associated in the sky forming rain causing clouds and temperature and atmospheric pressure ignite the clouds to drizzle and rain due to gravity. The same process happens in human eyes.

I put forth once again strongly that the lachrymal gland secrets only the vital compounds carried by the cellular water to protect the eyes and forms the tear film.

\section{(ii) Nasal Fluid}

In this context it was worth clearing another myth behind nasal watering. Nasal is the part where plenty of Oxygen is always available as human being breath through nasal pipe. When Hydrogen molecules were released through nasal tissues around the nasal pipe, those molecules obviously bond with the Oxygen molecules and nose drains with watery fluid. Tears sometimes passes through a small opening on the inner side 
of the eyelid into the nose. But the rate of Hydrogen molecules pumping must be far less when compared to tissues surrounding the eye region. It is important to mention nasal fluid does not include filtration, as does the formation of urine by the glomerular kidney (source: http://www.aquaticape.org/tears.html).

\section{(iii) Perspiration Mechanism}

I further clearly substantiate with another process called "Perspiration" (Mosher, H.H., 1933). Due to lack of stable Oxygen molecules in the blood human tissues receive the at most pressure and this led to release of Hydrogen molecules through skin pores in a line. Chemical bonding that arises from the mutual attraction of oppositely charged Hydrogen and Oxygen molecules. Therefore, Oxygen molecules did not usually exist on own, but will bind with ions of opposite charge to form a water molecule from the atmosphere. Each drop coalesces with adjoining sweat drop leading to increase in drop size and the volume of sweating.

\section{Conclusion}

This theory is going to disprove hundreds of years old biochemical tears theories and sweat theories (William H. Frey II, 1985; Benjamin Milder, M.D. 1987) and ought to cease the thousands years of cry on cry. The scientists would realize their wrong projections since long years and realize now that tears were not secreted by lachrymal glands and perspiration was not due to sweat glands. Tears obviously contain some of the products along with cellular water as tear droplets were associated with tear film. Darwin reported in his book "Expression of the Emotions in Man and Animals" (Darwin, C., 1872) where observations of recently captured elephants showed no tearing and "the native hunters asserted they had never observed elephants weeping." I am to contradict his views as release of the Hydrogen molecules create the tears and any animal like Elephant, Whales, Monkeys etc., could develop tears.

I can add many more findings associated with my discovery like closing of eyes stops the contact with atmospheric Oxygen and tear formation gradually reduces. The fire lit before the crying baby limits the availability of atmospheric Oxygen and the baby stops crying. The dead bodies were advised to burn for the reason it would limit the atmospheric Oxygen availability stopping the sobering kin and kith at grave yard. My list is pretty long and I would mention in my next paper.

* Scientist (Ministry of Textiles)

\section{Literatures:-}

[1]. Finkel T, Holbrook NJ. Oxidants, oxidative stress and the biology of ageing. Nature. 2000; 408:239-47.

[2]. Nic, M.; Jirat, J.; Kosata, B., eds., "spectroscopy". IUPAC Compendium of Chemical Terminology (Online ed.). 2006; doi:10.1351/goldbook. \{file\}. ISBN 0-9678550-9-8).

[3]. William H. Frey II, Ph.D, , The Mystery of Tears Muriel Langseth. Minneapolis: 1985; Winston Press.

[4]. Darwin, C. (1872). The Expression of Emotions in Man and Animals. 1979; London: Julian Friedman Publishers.

[5]. Greenwood, Norman N.; Earnshaw, Alan Chemistry of the Elements (2nd ed.).Butterworth-Heinemann, 1997; ISBN 0080379419.,p. 28.

[6]. Lide, David R. "Section 4, Properties of the Elements and Inorganic Compounds; Melting, boiling, and critical temperatures of the elements". CRC Handbook of Chemistry and Physics (84th ed.). Boca Raton, Florida: CRC Press. 2003; ISBN 0-8493-0595-0.

[7]. Mosher HH (1933). "Simultaneous Study of Constituents of Urine and Perspiration". The Journal of Biological Chemistry 99 (3): 781 790.

[8]. Wu K, Galina VJ, da Costa SR, Sou E, Schechter JE, HammAlvarez SF. Molecular mechanisms of lacrimal acinar secretory vesicle exocytosis. Exp Eye Res 200683 84-96. 